\title{
Remote memory access: A case for portable, efficient and library independent parallel programming
}

\author{
Alexandros V. Gerbessiotis* and Seung-Yeop Lee \\ CS Department, New Jersey Institute of Technology, Newark, NJ 07102, USA
}

\begin{abstract}
In this work we make a strong case for remote memory access (RMA) as the effective way to program a parallel computer by proposing a framework that supports RMA in a library independent, simple and intuitive way. If one uses our approach the parallel code one writes will run transparently under MPI-2 enabled libraries but also bulk-synchronous parallel libraries. The advantage of using RMA is code simplicity, reduced programming complexity, and increased efficiency. We support the latter claims by implementing under this framework a collection of benchmark programs consisting of a communication and synchronization performance assessment program, a dense matrix multiplication algorithm, and two variants of a parallel radixsort algorithm and examine their performance on a LINUX-based PC cluster under three different RMA enabled libraries: LAM MPI, BSPlib, and PUB. We conclude that implementations of such parallel algorithms using RMA communication primitives lead to code that is as efficient as the message-passing equivalent code and in the case of radix-sort substantially more efficient. In addition our work can be used as a comparative study of the relevant capabilities of the three libraries.
\end{abstract}

\section{Introduction}

In the past years several parallel computing models have been proposed such as the CGM [7], LogP [6], BSP [24], and QSM [15] for the design of parallel algorithms and the programming of parallel computers. At the same time a number of parallel libraries have become available that allow portable programming on a variety of parallel hardware platforms. Most of the these libraries are totally independent of these programming models; libraries based on the Message Passing Interface (MPI) such as the freely available LAM MPI [17] and MPICH [22], or commerical ones such as WMPI [5], and other libraries such as the Parallel Virtual Machine (PVM) [9] fall into this category all of which offer extensive library features of several hundred function calls. Other libraries such as the Oxford BSP library [21], BSPlib, The Oxford BSP Toolset [16, 23], and PUB-Library [2] (Paderborn University BSP-

\footnotetext{
*Corresponding author. E-mail: alexg@cs.njit.edu.
}

Library) are tied to a specific programming model and are quite compact thus offering a mere 30-40 function calls.

Parallel programming is still viewed as a complex task not only because it involves understanding and resolving issues such as task parallelism and data distribution but also because it requires effective processor communication and synchronization. Users of popular parallel programming libraries such as the ones based on the Message Passing Interface (MPI) are offered an extensive collection of message passing alternatives to realize two-way interprocessor communication. For example sending information under MPI can be performed by no less than eight different blocking or non-blocking function calls. Receipt of information can be dealt with by a variety of approaches as well that also depends on the sending method used. The choice of multiple methods to perform a single task leads many times to programmer overload and confusion, increasing programming complexity, deadlocked programs, and results in program inefficiencies as the most efficient way to communicate information may 
not be used. MPI for example offers the programmer more than 200 library functions even though most programmers use only a small subset of these functions.

Remote memory access (RMA) one-sided communication has been available since the introduction of the Cray SHMEM routines [4]. Because of the simplicity of these routines, their elegance and their efficiency, RMA was quickly and successfully adapted into bulk-synchronous parallel libraries such as the Oxford BSP library, BSPlib, and PUB-Library. Subsequently it was incorporated into MPI under the MPI-2 standard. The current support of RMA under MPI libraries varies, however. Some freely available libraries such as LAM MPI support it but others do not. Because of this nonuniformity of support programmers stay away from this style of parallel programming because, it is thought, it could lead to non-portable code. Although a programmer is offered at least eight ways to send information through message passing in MPI, there is only one way to send information through RMA by utilizing a put instruction; a symmetric get instruction is also available. In BSPlib and PUB-Library, the programmer also has two choices for a put (or get).

In this paper we make a strong case for remote memory access as an effective way to program a parallel computer. We support our case by first proposing a robust, simple, intuitive, and extensible programmatic framework that supports RMA in a library independent, simple, and portable way using the $\mathrm{C}$ programming language; extending this framework for $\mathrm{C}++$ and Fortran programming would be easy. We claim that if one uses our approach the parallel code one writes will run transparently not only under MPI-2 RMA enabled libraries including LAM MPI and Critical Software's WMPI, but also on bulk-synchronous parallel libraries such as BSPlib and PUB-Library thus making one's code library independent as well. Future libraries that support similar APIs could also be accommodated. We claim that the advantage of using RMA is code cleanliness, reduced programmer confusion and overload and increased code efficiency because two-sided communication is much more tedious than one-sided communication. In addition, the programmer does not need to choose the best way for interprocessor communication as there is only one method to do so. Moreover, a communication library implementor can more easily provide an efficient implementation of such a method, rather than optimize eight or more apparently equivalent ones.

We support our case by implementing using the proposed framework three sets of benchmark quality par- allel programs using one-sided communication: (a) a set of tests that assess the communication and synchronization performance of a parallel hardware platform that will thereafter be referred to as the As suite, (b) a set of parallel dense matrix multiplication programs, the Mult suite, and (c) a set of parallel integer radixsort programs, the Rdx suite. We then examine the performance of these programs on a LINUX-based PC cluster under three different RMA enabled libraries: LAM MPI, BSPlib and PUB-Library. For comparative purposes variants of these programs have been made to work using two-sided communication in LAM MPI. We then compare the performance of these programs under one-sided and two-sided communication in LAM MPI, and also compare the one-sided communication performance under BSPlib and PUB-Library to the one-sided and two-sided communication performance of the same programs under LAM MPI.

Through the As suite, we can test the synchronization capabilities of a parallel platform under each one of the three libraries. We can also test the communication capabilities of a parallel platform under any of these libraries by benchmarking total-exchanges implemented by using a put operation in all these libraries and through a variety of send operations in LAM MPI. In the Mult suite, we implemented a blocked parallel dense matrix multiplication algorithm using one-sided put or get functions calls in all three libraries and in addition, two-sided send/receive library calls in LAM MPI. Finally the Rdx suite, implements two integer radix-sort algorithms. The first algorithm is a straightforward parallelization of ordinary radix-sort for 32-bit integers. An optimized version of this algorithm that allows for round-robin key value-based delayed communication was also implemented. The second algorithm is an enhanced version of the straightforward parallelization where messages are combined before being sent out so that each processor sends and receives one long message.

We carry out this experimental study for one additional reason: it offers a comparison of the communication performance of the three libraries on a cluster of PC workstations under a realistic set of benchmark programs. In that respect, our work serves similar but more general objectives than the work of [8] that compares SHMEM and MPI functionality and [18-20] that evaluate MPI-2 one-sided functions on a variety of high performance parallel machines.

Our conclusions seem to consistently confirm our claims. One-sided communication is easier to deal with than two-sided communication in parallel programs. 
BSPlib and PUB-Library have no more than 30-40 library calls and seem to do as good a job as a more general library such as LAM MPI. In the case of LAM MPI, the single choice of a one-sided function call is always the best or as good a choice as the best of a collection of two-sided alternatives. In complex communication patterns that resemble total-exchanges, where each processor sends all other processors multiple messages, one-sided communication seems to outperform two-sided communication at least in LAM MPI. Onesided communication as implemented in BSPlib seems to be better than two-sided and also one-sided communication under LAM MPI, with some minor exceptions. Even one-sided communication in PUB-Library, for the cases that the library seems to be properly tuned, outperforms one-sided or two-sided LAM MPI communication. MPI in general and LAM MPI in particular needs to be downsized into a smaller set of library calls that are more optimized and fine tuned and give less freedom of choice to the average programmer.

\section{A framework for parallel programming}

In this section we show how one could write parallel code using remote memory access one-sided communication that is both portable among several parallel platforms but also, library independent in the sense that the same program can be compiled without any rewriting under RMA enabled libraries which include MPI-2 based libraries such as LAM MPI and Critical Software's WMPI, and programming model specific libraries such as BSPlib and PUB-Library. Towards this we introduce a few generic primitives that one could use for such portable and library independent parallel programming. For the most important of these primitives equivalent MPI-2 translations are given as well as BSPlib/PUB translations that adhere to the BSPWorldwide standard [16].

All three libraries considered in this study have similar methods for initializing an SPMD parallel program, terminating it, aborting it, and accessing the number of processors and the processor identification number of an individual processor. In our framework we use the generic wrapper functions LIBEGIN(LINPROCS()), LIEND(), LIABORT(), LINPROCS(), and LIPID() to represent these common functions. Such functions can be mapped easily to individual library function calls. We omit this by noting that the source code of the programs written for this study contains a file named ai.h that includes detailed mappings for these functions in all three libraries. For the sake of an example, in LAM MPI, the first of the generic primitives maps for example to an MPI_Init (the LINPROCS() argument is an idiosyncratic feature of BSPlib that is ignored), the second to an MPI_Finalize, the third to an MPI_Abort, the fourth to an MPI_Comm_size, and the last one to an $M P I \_C o m m \_r a n k$ function call.

We provide two ways for RMA-based programming that we explain in detail below. Suppose that source processor spid intends to send information to a destination processor $d p i d$. The source information is stored in consecutive memory locations starting at address $s r$ $c a d d r$ and is len bytes long. The information is to be stored in processor $d p i d$ starting at an offset of off bytes from the starting address desaddr. This operation can be realized by issuing a put instruction at spid that has the following format: LIPUT(dpid, srcaddr, desaddr, off, len). One other reason for proposing our framework is to simplify the API of the one-sided MPI primitives; for example the put and get instructions in MPI-2 have a counter-intuitive syntax.

Certain actions must precede this operation and the operation is guaranteed completion by a second set of actions that take place after the LIPUT is issued. The actions preceding the operation involve registration of the destination desaddr and the amount of information len that will be transferred. Such actions may involve exchange of information among the processors that participate in the communication. Such registration is split into three steps: (a) a registration call LIREGISTER(desaddr,len), followed (b) by a communication

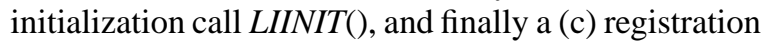
commit call LICOMMIT(). A registration commit call is provided because registration may require the exchange of some information before actual communication takes place. A communication initialization call is provided to accommodate for example asynchronous, or more specifically, non bulk-synchronous parallel libraries. Therefore, not all three call are always needed. The communication initialization call can be located well-after the registration call as long as it precedes the actual communication call LIPUT. After the LIPUT has been issued, communication is realized by issuing a communication completion call using an LISYNC() instruction and concluding the communication by a barrier call LIBARRIER().

This common interface that we provide to the three libraries may look similar to some to the one agreed in [16] for a common programmatic interface for onesided communication. There are, however, some differences intended to support the MPI-2 one-sided communication interface. 


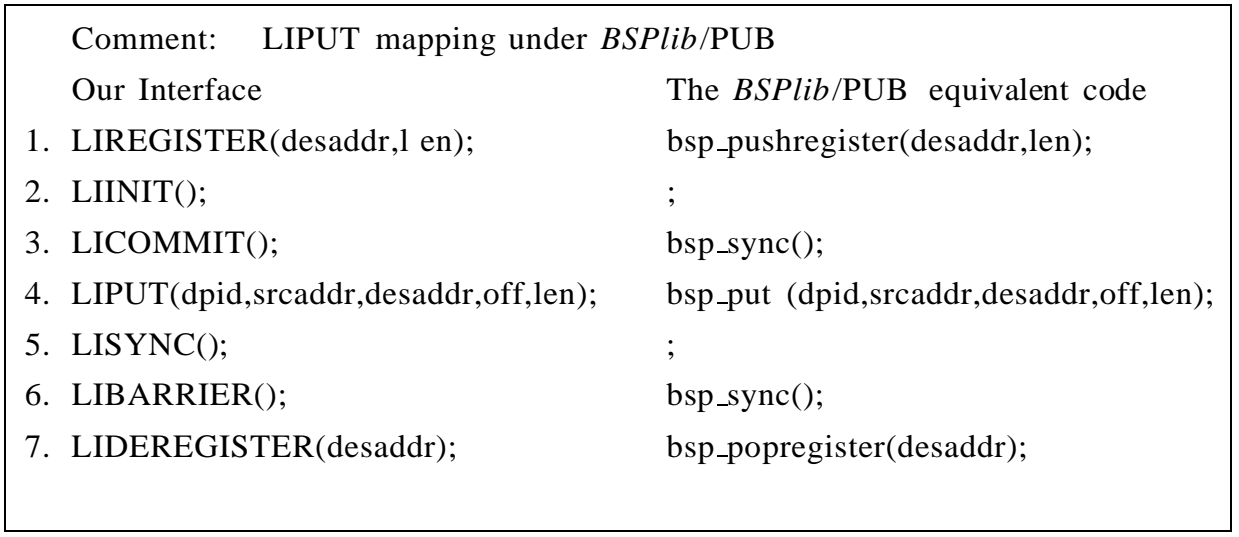

Fig. 1. An LIPUT mapping under BSPlib/PUB.

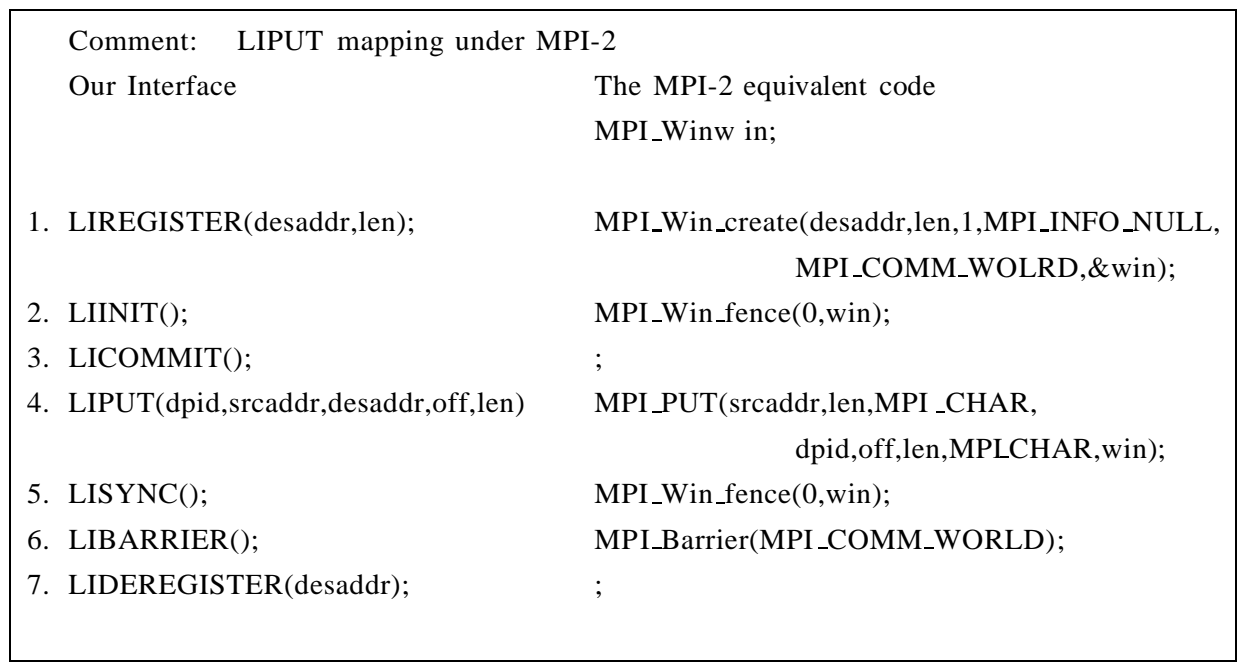

Fig. 2. An LIPUT mapping under MPI-2.

We show the mapping of our proposed interface to the one supported by both BSPlib and PUB-Library for the example above in Fig. 1 and to the one supported by LAM MPI in Fig 2.

With reference to Fig. 2 we note that RMA operations under MPI-2 are non-blocking. The MPI_Win fence call is one of three different mechanisms for marking completion of a one-sided communication in MPI-2. The instruction of line 2 opens a window for communication and closes it by effecting the communication of line 4 by a similar instruction in line 5 . A fence is essentially a barrier synchronization, similar to the Cray SHMEM routine shmembarrier. Therefore LIBARRIER may map to a null instruction in such a case; yet the equivalence under the BSP model, if one insists on it, of the two mappings of LIPUT under BSPlib/PUB and MPI-2 still remains. In all cases, reg- istration becomes effective after the commit call in line 3.

The introduced example assumes that only one active window is present. In BSPlib and PUB-Library, all communication within one superstep (segment of computation that can be completed using locally available information) can be associated with a single such window. Under LAM MPI, however, if two communication operations are performed these must be registered and realized sequentially one after the other to a single window or registered to different windows. For this to occur we need to be able to map distinct destination addresses to distinct windows. One way to do this is by what we call "labeled windows" and the mapping of our generic framework to the two sets of libraries is depicted in Figs 3 and 4. 


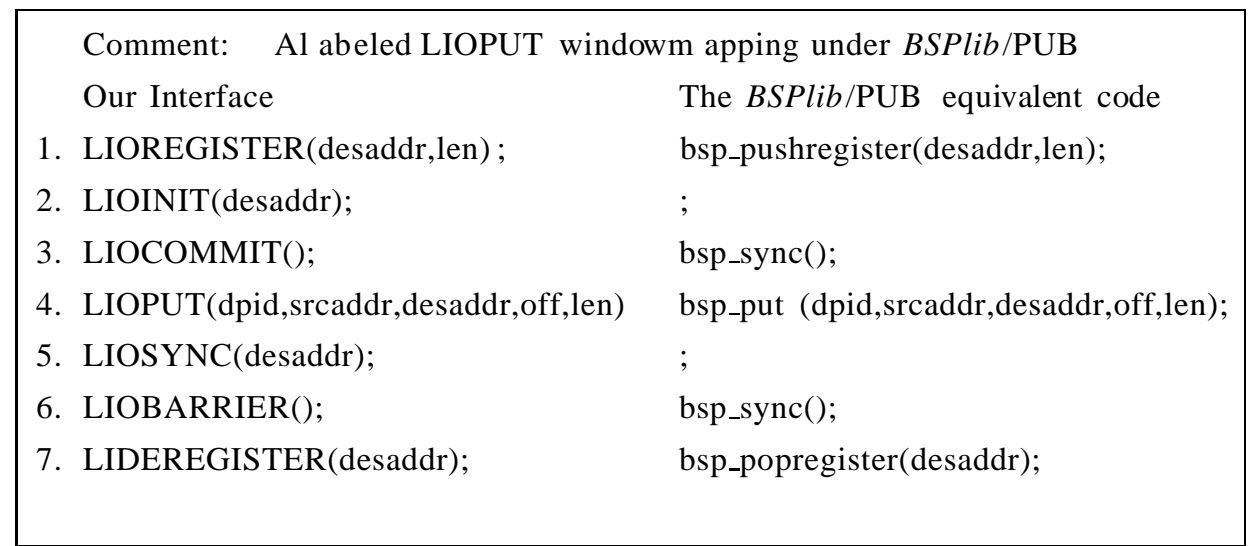

Fig. 3. A labeled LIOPUT window mapping under BSPlib/PUB.

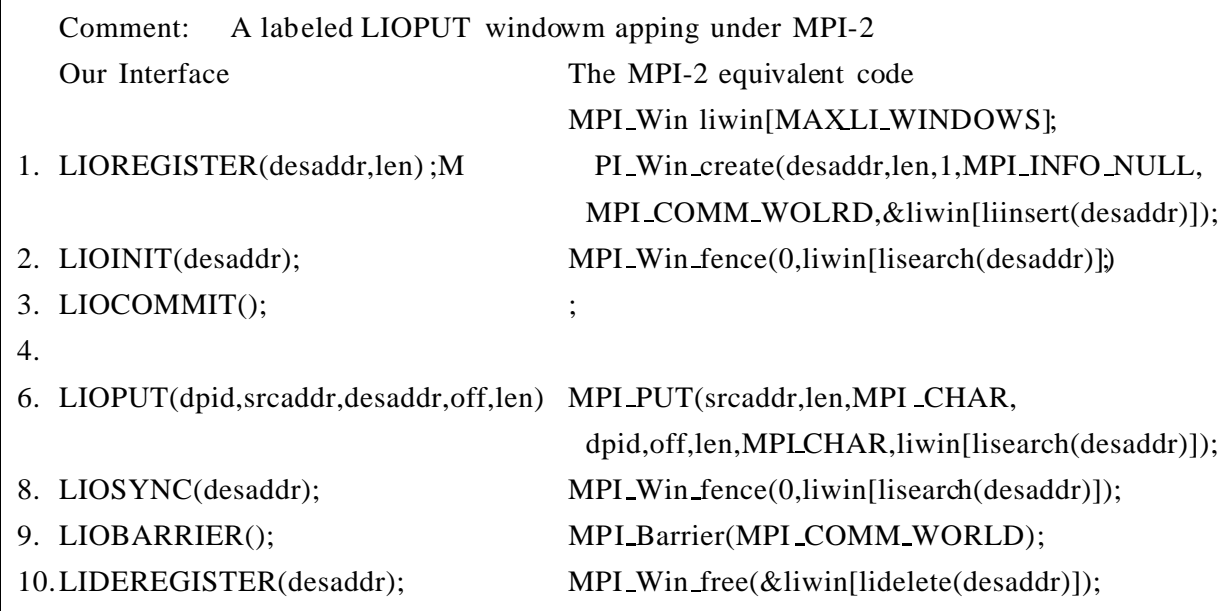

Fig. 4. LIOPUT under MPI-2.

As it is shown in Fig. 3 the mapping does not change for the pair of BSPlib/PUB if we use "labeled windows". It is more involved in Fig. 4 for MPI-2. We first assume that we have elsewhere (eg. initialization of the SPMD program) allocated an array liwin of communication windows of sufficient dimension. Each desaddr must uniquely identify such a window by implementing functions liinsert(desaddr), lisearch(desaddr) and lidelete (desaddr) that create a unique window index, search for the unique window index of address desaddr, and free the unique window index associated with desaddr. Other than that Fig. 4 is similar to Fig. 2. The mapping for LIGET and LIOGET primitives under the two sets of libraries is defined similarly.

With the aforementioned simple common mappings of the different methods employed for one-sided communication in the three libraries, one is able to use a common interface and thus write code that is library independent and works on all three libraries. We have employed this approach not only in developing the code that is described in this paper but also in other work; such code is available at the first author's Web-page.

\section{The As suite}

The As suite tests the synchronization capabilities of a parallel platform under any library that supports or can be made to support our RMA framework. It also tests the communication capabilities of a parallel platform for a tested communication library by measuring the realization of total-exchanges (also known as $p$-relations). 
The first set of tests in this suite measures latency related performance of the communication library including the cost of barrier synchronization. The tests in this suite are described below.

(1) Test 1 (Empty). This test times a barrier synchronization if it is available in the communication library.

(2) Test 2 (Comp). This test times a barrier synchronization that is preceded by an elementary computational operation; the purpose is to assess what the effect of such a computational operation will be to the time reported by Test 1 .

(3) Test 3 (Full). This is the time it takes to perform a total-exchange of $p$ integers followed by a barrier synchronization, where $p$ is the number of available processors. Thus each processor sends and receives $p$ integers $(p-1$ from remote processors and one integer through a local copy/communication-less operation). The purpose of this test is to assess what the effect of a simple communication operation that involves all processors will be to the time reported by Test 1.

(4) Test 4 (Simple). This is the time it takes to perform a simple communication of one integer between processor 0 (sender) and processor $p-1$ (receiver) followed by a barrier synchronization. The purpose of this test is to assess what the effect of a very simple communication operation that involves the least number of processors will be to the time reported by Test 1 .

(5) Test 5 (Scatter). This is the time it takes for processor 0 to scatter one integer to each of the remaining $p-1$ processors, followed by a barrier synchronization. The purpose of this test is to compare the time of this test to the timing results reported by Test 3 and Test 4 .

These tests can be useful to someone who wants to assess the cost of barrier synchronization and understanding the latency behavior of the combination of the parallel hardware platform and the communication library tested. In addition, these tests can be useful to those who are interested in deriving the bulk-synchronous parallel model characteristics of the hardware platform. We note that in the BSP model [24] a parallel platform is modeled by a tuple $(p, L, g)$, where $p$ is the number of processors, $L$ is the synchronization periodicity, and $g$ the cost of communication per word of information (inverse of router throughput). More formally $g$ is defined so that the cost of realizing an $h$-relation (an in- stance of communication where each processor sends and receives at most $h$ words) in continuous message usage (for large $h$ ) is $g h$; for smaller values of $h$ the cost of communication is latency bound by $L$. Thus these tests can help us determining the value of $L$.

Finally, the As suite includes tests that measure the communication performance of the parallel hardware and the communication library under realistic assumptions. We measure such performance by timing totalexchanges where each processor communicates a total of $h$ integers to every other processor (including itself) for increasing value of $h$. If the communication time for such an operation is $t$, the cost of communication is considered to be $t / h$. We perform this test for $h$ that is a power of two starting from a value equal to the number of available processors and doubling it after every completed test. Under all three libraries used in this work the total-exchange is realized by performing a series of put instructions. In addition, in LAM MPI we realize the total-exchange by using two-sided communication send/receive instructions. Among the variety of such send/receive combinations available in MPI we timed and report the one that offered the best overall performance; this was the MPIIrecv and MPI_Send combination. Thus we are able to compare one-sided and two-sided communication in LAM MPI and the one-sided communication performance of the other libraries to the one-sided/two-sided performance of LAM MPI. We note that total exchanges under LAM MPI could have been implemented by using MPI collective communication primitives; these, however, provided no better performance than the chosen direct implementations.

The programming language used in the implementations is ANSI C and the code was tested for scalability and portability on a cluster of 16 dual-processor PC workstations (PentiumII 350 Mhz, Redhat Linux 7.1running, $128 \mathrm{MB}$ RAM equipped) with communication performed through 100Mbit 3Com-905B Ethernet cards and a 24-port CISCO Catalyst 2924 M-XL-EN switch connecting the workstations. The default GNU Project's gcc compiler is used through the LAM MPI, BSPlib, and PUB-Library front-ends, and the sourcecode is compiled with the -O3 compiler option set. Timing is obtained through the use of real-time wallclock time. All results and variables used for communication and computation involve ANSI C int 32-bit data types. The code developed for this experimental study is publically available at the first author's Webpage [14]. The tested platform is either the 2-node, 4-node, or 16-node PC cluster utilizing only one CPU 
per node (non-SMP configuration) or both CPUs (SMP configuration). By setting up the cluster this way we also examined the effects of symmetric multiprocessing in parallel programming. Version 1.4 of BSPlib, the default version of LAM MPI for Red Hat Linux 7.1 (LAM 6.5.1), and PUB-Library version 8.0 were used in the experiments.

Figure 5 shows the synchronization performance of the PC cluster. It seems that LAM MPI gives the worst results in SMP and non SMP configurations. PUBLibrary is better than BSPlib in Test 3, and in all other tests it is worse or in some cases equivalent (Test 5 , non SMP configuration) to BSPlib. One interesting observation is that Test 1 , Test 2 , Test 4 , and Test 5 of BSPlib are better than those of the other two libraries. The performance of Test 3 in BSPlib, and the one that matters most, is worse than that of PUB-Library although better than that of LAM MPI.

Communication performance varies with $p$. With reference to Fig. 5 (detailed tables with numeric figures for the various points of the graphs can be found in [10]), for $p=4$ and $p=8$, PUB-Library seems to be consistently better than BSPlib and LAM MPI is slightly worse than the other two if its send performance is considered; the put based performance seems to be slightly worse. For $p=16$ and $p=32$, however, BSPlib is more scalable and gives better results but in several cases this requires that the programmer set appropriate values to some communication control variables of BSPlib. LAM MPI is close second but is much better tuned, consistent and predictable. The performance of the PUB-Library quite surprisingly is significantly worse than the other two in both SMP and non SMP configurations. In addition, it seems that the PUB-Library's performance deteriorates for a range of $h$ that varies between $2 \mathrm{~K}$ and $16 \mathrm{~K}$; for larger values it is still at least a factor of two worse than the other two libraries. We note that for $h=4 K$ PUB-Library exhibits an observable loss of performance which may be related to some kind of poor tuning. We note that some tests were not carried over because the corresponding configurations were unavailable (eg. 32-processor nonSMP configuration).

The As suite offers us a first glimpse on the performance of the three libraries in the PC cluster of our experiments. Concluding, we can say that one-sided communication in BSPlib outperforms one-sided or twosided communication under LAM MPI; the same can be said for PUB-Library but for small $h$ only (less than $4 \mathrm{k}$ ). Under LAM MPI two-sided communication is preferable to one-sided one; this is due, however, to the fact that the latter is built on top of the former. The benchmarks of the As suite are complemented by the two suites Mult and Rdx that are described in the following sections.

\section{The Mult suite}

The Mult suite contains various implementations of a parallel dense matrix multiplication algorithm that is optimal in computation, it is memory efficient but is neither synchronization nor communication efficient. It exhibits, however, satisfactory computational performance.

A precursor to this algorithm is one that was originally described in [24] in the context of the BSP model of computation. Let two matrices $A$ and $B$ of dimension $n \times n$ be distributed evenly among the $p=\sqrt{p} \times \sqrt{p}$ processors of a parallel computer so that each processor gets a contiguous submatrix of dimension $n / \sqrt{p} \times n / \sqrt{p}$ of $A$ and $B$ and will also compute one identically dimensioned submatrix of the product $C$ of $A$ and $B$. Let $P=\sqrt{p}$ and $N=n / P$; the algorithm assumes that $p \leqslant n^{2}$. In the algorithm described in [24], each processor $m$ in a single round of communication obtains all the required data consisting of $P$ blocks of $A$ and $B$ and computes a result associated with a submatrix of $C$. The resulting algorithm is neither memory nor communication efficient; for example each processor requires local memory enough to accommodate $2 P$ blocks of dimension $N \times N$. We can convert this algorithm into a memory efficient version that only requires additional local memory for two such $N \times N$ blocks by performing the computation in $P$ rounds of communication so that in each such round a single $N \times N$ block of $A$ and $B$ is communicated and a partial result of a block of $C$ is computed. This algorithm [13] is a modification of Cannon's method and depicted below in Fig. 7 for processor pid. All matrices are stored in a column major format in a one-dimensional array; element $A[i, j]$ is thus $A[j * n+i]$. The communication of lines 5 and 6 can be implemented by put or send instructions. Lines 5 and 6 guarantee that each processor sends two $N \times N$ and receives two $N \times N$ blocks into $a$ and $b$ that will be required for matrix multiplication. Because of the "pipelined" fashion of the communication, every processor receives the required blocks in line 7 that will be used in the multiplication of line 9 . In line $8, a$ is transposed into $a^{t}$ to optimize the computation and take advantage of the column oriented storage of $a$ and 

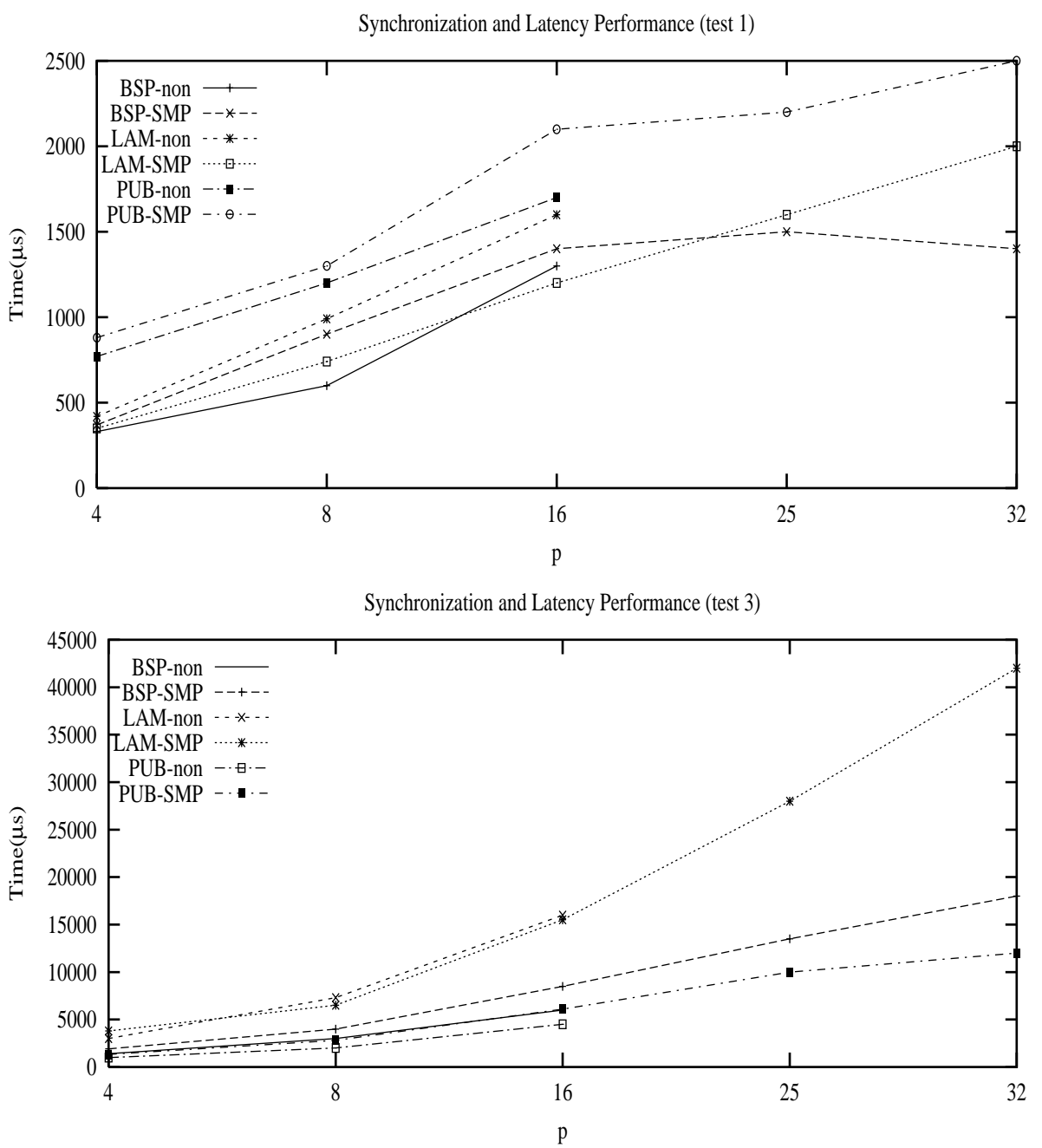

Fig. 5. Synchronization and Latency performance of LAM MPI, BSPlib, and PUB.

$b$. In the standard loop of Cannon's algorithm, rows of $a$ would be multiplied with columns of $b$. Since we store $a, b$ in column major orientation, if we transpose $a$ before the multiplication, then the multiplication will involve columns of $a^{t}$ and $b$. This requires a reorientation of Cannon's matrix multiplication loop. Figure 8 shows how the "pipelined" just in time delivery of $a, b$ can be performed by using get operations instead.

We implemented MatMul by using put instructions and MatMulG using get instructions in all three libraries. In addition, in the case of LAM MPI, we implemented MatMul using MPI_Irecv and MPI_Send two-sided communication instructions. We performed the experiments in non SMP configurations for $p=4$ and $p=16$ and on SMP configurations for $p=4$, $p=16$, and $p=25$. Given that dense matrix multiplication is a communication intensive operation the SMP experiments are expected to stress the communication network and show whether such matrix operations are suitable for SMP clusters as well. The methodology of the As suite was also followed in writing, compiling and executing the Mult tests noting that the involved arrays are ANSI C double data type aggregates.

Table 1 contains speedup results for $p=4,16$ processors in a non-SMP configuration of the cluster, and SMP configurations of $p=4,16,25$ processors. We also include sequential running time results for $p=1$; timing results for various processor configurations can be obtained from these and the included speedup figures. We kept problem size small as for larger problem sizes caching effects provide superlinear speedups. However the relative performance of the algorithms under the various communication mechanisms is still that obtained from the problem sizes depicted. 

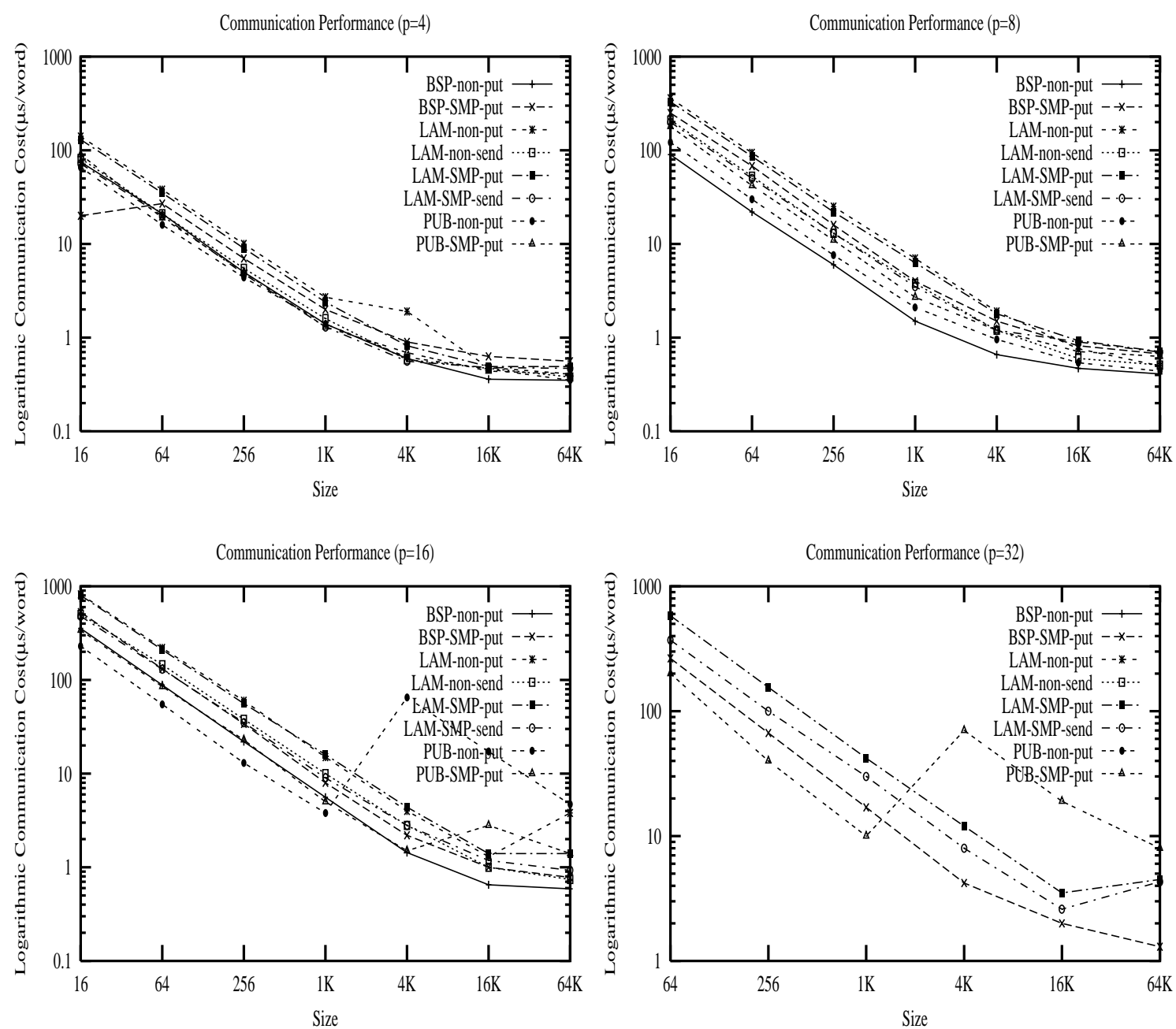

Fig. 6. Communication performance ( $\mu$ s/32-bit word) of LAM MPI, BSPlib, and PUB.

In the non-SMP configuration, for $p=4$, the communication performance of the three libraries as tested in the As suite is reflected in the speedup results we obtained. PUB-Library and LAM MPI have almost the same performance, with BSPlib a close second for the put implementations. In get implementations LAM MPI is superior to PUB-Library, which in turn outperforms BSPlib. An interesting observation is that in the PUB-Library and BSPlib there is an asymmetry in the performance of the put and get implementations. In LAM MPI, however, the two exhibit almost the same performance. For $p=16$, BSPlib seems to be more scalable, and has the best performance of the three, with PUB-Library a close second and LAM MPI an even closer third. The get implementation in BSPlib seems to be faster than the other two except for small $p$, where LAM MPI is better. It seems that the library latency of LAM MPI is higher than the other two. The put implementation in LAM MPI seems to be as fast as the send or get implementations.

In the SMP configuration, for $p=4$, and the put implementation LAM MPI and PUB-Library have a slight advantage over BSPlib and over the send implementation in LAM MPI. The get implementation in LAM MPI again is as good as the put. The get implementation of the other two libraries is much worse than the put or the LAM MPI get implementation. Similarly to the non-SMP configuration, for $p=16$ and $p=25$ processors BSPlib seems to have an advantage over the other two libraries for the put implementation. Surprisingly, however, the get implementation in LAM MPI seems to fare better than the PUB-Library and BSPlib for small $p$. The send implementation in LAM MPI is just a little faster than the put and get LAM 


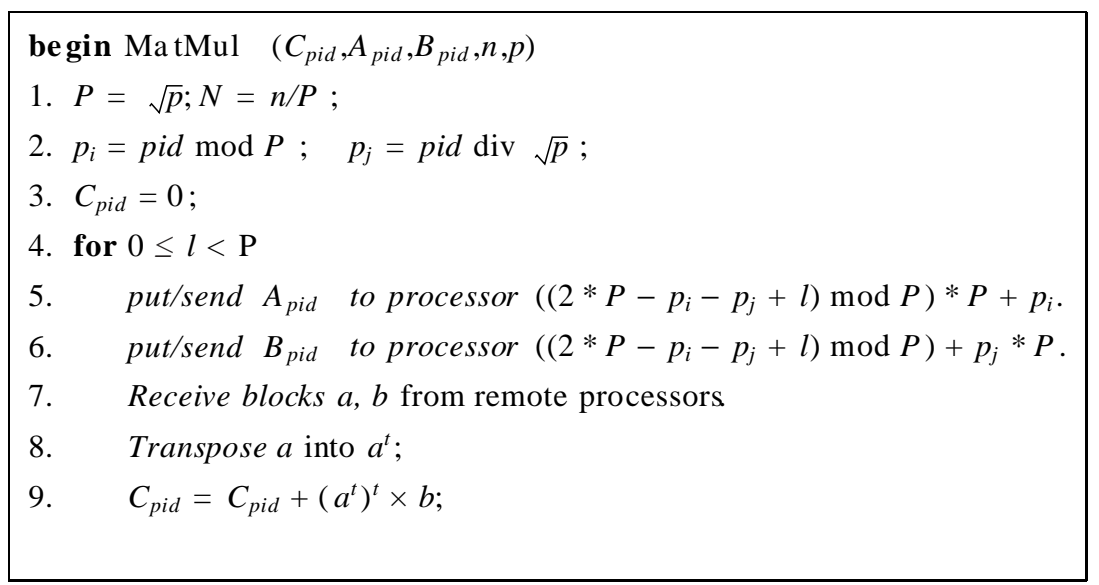

Fig. 7. Procedure MatMul.

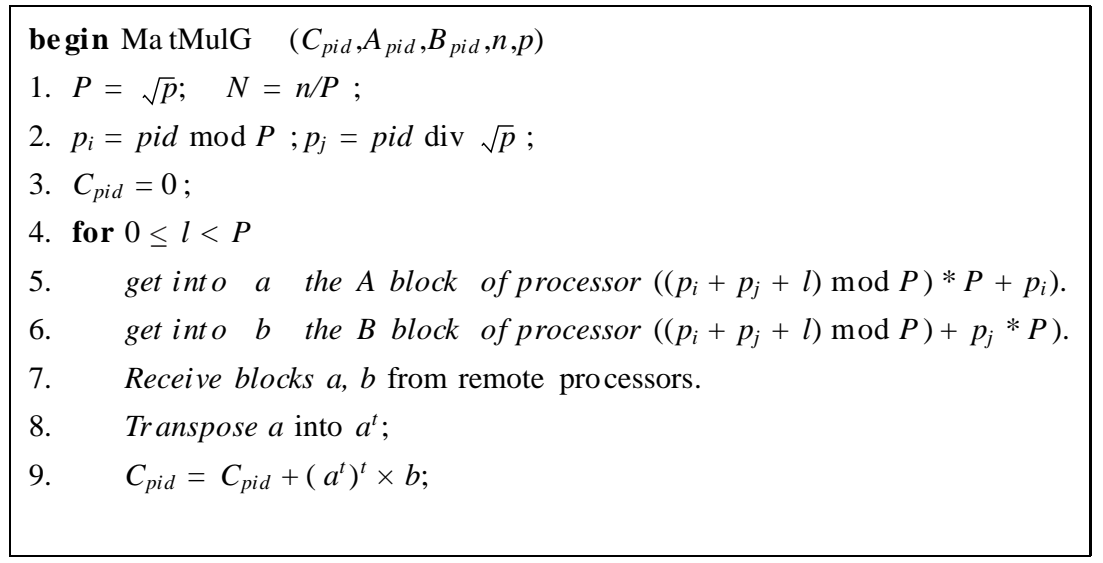

Fig. 8. Procedure MatMulG.

MPI implementations but slightly slower than the put implementations of BSPlib and PUB-Library.

Peak performance-wise BSPlib and PUB-Library look better than LAM MPI. One-sided communication (mostly put, less often get and rarely both) seems to consistently outperform two-sided communication. Even LAM MPI one-sided communication seems to fare well compared to its two-sided counterpart. Consistency-wise ( put vs get for example) LAM MPI is solid, in the other two libraries put is preferable to a get. As a side note, if we had not performed the transposition in line 8 of Fig. 7 or Fig. 8 with the corresponding change of line 9 , then the performance results we report would have been three to seven times worse compared to the reported ones. The SMP configurations gave satisfactory results compared to the non-SMP configurations thus indicating the suitability of dense matrix computations for SMP clusters.
The low speedup figures for $n=320$ are quite natural and easily explained through the As derived figures. Sequential matrix multiplication performs $2 n^{2}(n-1)$ floating point operations. For $n=320$ and the running time of 0.93 seconds this translates roughly to $1 / 70$ microseconds per floating point operation. Let $f=1 / 70$. The sequential time is thus $2 n^{2}(n-1) f$. The parallel time of algorithm MatMul and MatMulG is $2 n^{2}(n-1) f / p$ for computation and $2\left(n^{2} / p\right) \sqrt{p} g$ for communication if $g$ is the cost in microseconds per double of a total-exchange of $2 n^{2} / p$ double data types (or $4 n^{2} / p$ int data types). The ratio of sequential running time to parallel running time gives the speedup of the parallel execution; this is $p /(1+\sqrt{p} g /(f(n-1)))$. For $p=16, n=320, f=1 / 70$ and $g$ twice the figure (as a double is a 64-bit quantity) read from Fig. 6 for an $h=4 n^{2} / p=25600$, we obtain that for the SMP and non-SMP configurations in BSPlib and LAM the 
Table 1

Matrix Multiplication in non-SMP and SMP configurations

\begin{tabular}{|c|c|c|c|c|c|c|c|c|}
\hline \multirow{2}{*}{$\begin{array}{c}\text { Size } \\
n\end{array}$} & \multirow{2}{*}{$\begin{array}{c}\text { Time } \\
p=1\end{array}$} & \multicolumn{2}{|c|}{ PUB } & \multicolumn{2}{|c|}{ BSPlib } & \multicolumn{3}{|c|}{ LAM MPI } \\
\hline & & Put & Get & Put & Get & Put & Get & Send \\
\hline & & \multicolumn{7}{|c|}{ Speedup for $p=4$ non-SMP } \\
\hline 320 & 0.93 & 4.23 & 4.04 & 4.23 & 4.04 & 4.43 & 3.88 & 4.43 \\
\hline \multirow[t]{2}{*}{640} & 7.67 & 6.45 & 5.64 & 6.50 & 5.22 & 6.67 & 6.67 & 6.50 \\
\hline & & \multicolumn{7}{|c|}{ Speedup for $p=16 \mathrm{SMP}$} \\
\hline 320 & 0.93 & 9.30 & 3.58 & 10.33 & 10.33 & 5.17 & 6.20 & 8.45 \\
\hline \multirow[t]{2}{*}{640} & 7.67 & 16.32 & 7.91 & 18.26 & 16.32 & 14.75 & 13.95 & 15.04 \\
\hline & & \multicolumn{7}{|c|}{ Speedup for $p=4 \mathrm{SMP}$} \\
\hline 320 & 0.93 & 3.58 & 3.44 & 4.65 & 3.32 & 5.17 & 4.43 & 4.89 \\
\hline 640 & 7.67 & 7.10 & 5.48 & 6.85 & 4.54 & 7.10 & 7.10 & 6.97 \\
\hline \multicolumn{9}{|c|}{ Speedup for $p=16 \mathrm{SMP}$} \\
\hline 320 & 0.93 & 6.64 & 5.17 & 7.75 & 7.15 & 5.47 & 5.17 & 6.64 \\
\hline 640 & 7.67 & 12.78 & 7.59 & 14.47 & 12.57 & 11.45 & 11.62 & 11.98 \\
\hline \multicolumn{9}{|c|}{ Speedup for $p=25 \mathrm{SMP}$} \\
\hline 320 & 0.93 & 7.75 & 3.44 & 8.45 & 9.30 & 4.43 & 2.16 & 7.15 \\
\hline 640 & 7.67 & 15.65 & 7.17 & 19.18 & 16.32 & 13.00 & 13.00 & 14.47 \\
\hline
\end{tabular}

corresponding speedup figures should be around 8 and 5 for the non-SMP put implementation and 6 and 4.6 for the SMP put implementation; the actual speedup figures are 10.33 and 5.17 for the former and 7.75 and 5.47 for the latter implementation.

\section{The RDx suite}

Finally, we have implemented and studied the performance of a parallel version of the sequential radixsort algorithm. As opposed to comparison-based sorting algorithms such as quicksort, mergesort, and heapsort that can sort any input keys, radix-sort and its base algorithm count-sort [3] can sort only keys that take fixed values by counting the occurrences of each key value. In radix-sort, we perform a number of rounds of the elementary count-sort algorithm [3]. If we view a 32-bit word as four radix-256 digits then radix-sort will require four rounds of a count-sort algorithm. If each key is viewed as two radix-65536 digits, then we will need two rounds of count-sort. For the test range of the experiments to be presented below, the four-round algorithm was faster than the two-round case in all sequential and parallel experiments and it is the one that was parallelized.

A brief description of the parallel radix-sort algorithm is sketched in Fig. 9. Parallel RDXSORT for 32bit unsigned integers consists of four rounds of parallel countsort PCountSort that works on distinct bits of the input keys starting from the least significant bits. Parallel countsort PCountSort calls the sequential count-sort algorithm in line 2, which counts the occurrences of each key, and uses this information in the parallel prefix (scan) step of line 3 to determine the final destination of each input key. Since as a byproduct of line 2 , the keys are sorted based on the values of the 8 bits under consideration, the routing of line 5 can be performed in blocked fashion for each value of the 8 bits.

An optimization was incorporated into this algorithm; the optimized algorithm is RDXSORTOPT shown in Fig. 10. RRDXORTOPT works similarly to RDXSORT except that the processors route their keys by considering distinct range values at a time. In line 4 , processor 0 still sends first the keys with value 0 ; however, processor 1 sends first the keys with value $256 / p$, where $p$ is the number of available processors. The intent of this optimization is two-fold: (a) separate/reduce concurrent message transmissions to the same destination, and (b) increase the time gap between service requests and thus the service time. Pid is the identification number of the processor executing the statement, and the term "wrappedto" means that if variable range reaches 255 before the value on the right side of "wrappedto", then the next value range takes is zero and subsequently range is incremented until the value to the right of the "wrappedto" is reached. At the end of the routing, an artificial delay in the form of a for loop of several microseconds is inserted in line 6 .

Among the three test suites we have examined, RDXSORT is the one that tests to the limit the communication performance of the hardware platform and the capabilities of the communication library used. In lines 4-5, each processor issues 256 communication requests to as many as $p$ processors. Timing results for $p=1$ and speedup results for non-SMP and SMP 


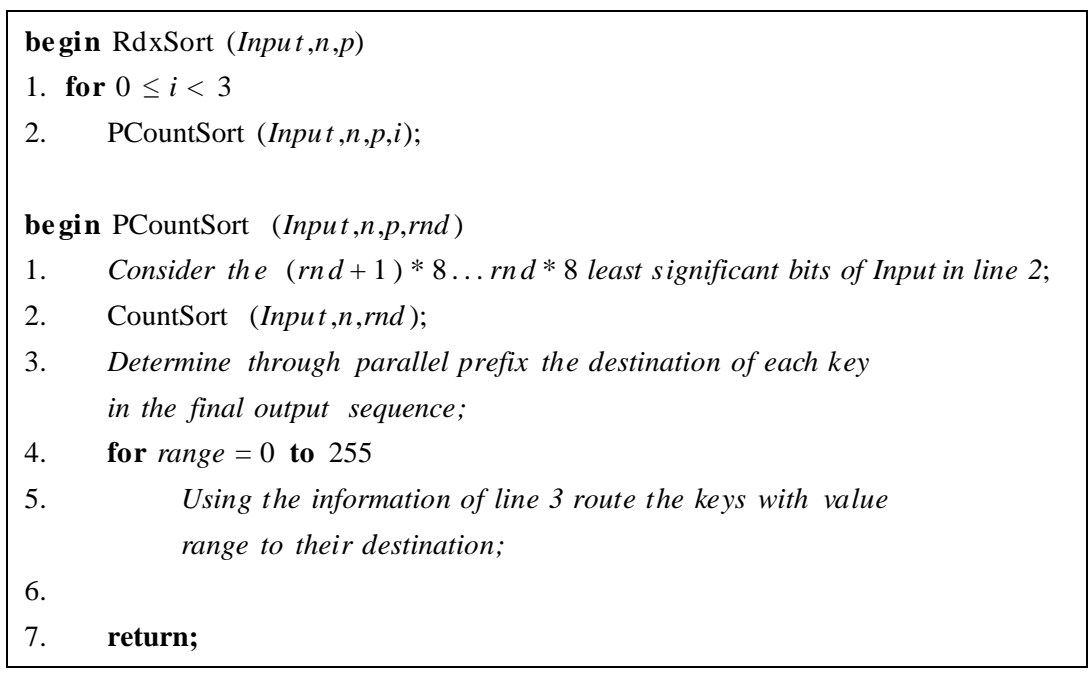

Fig. 9. Procedure RDXSORT.

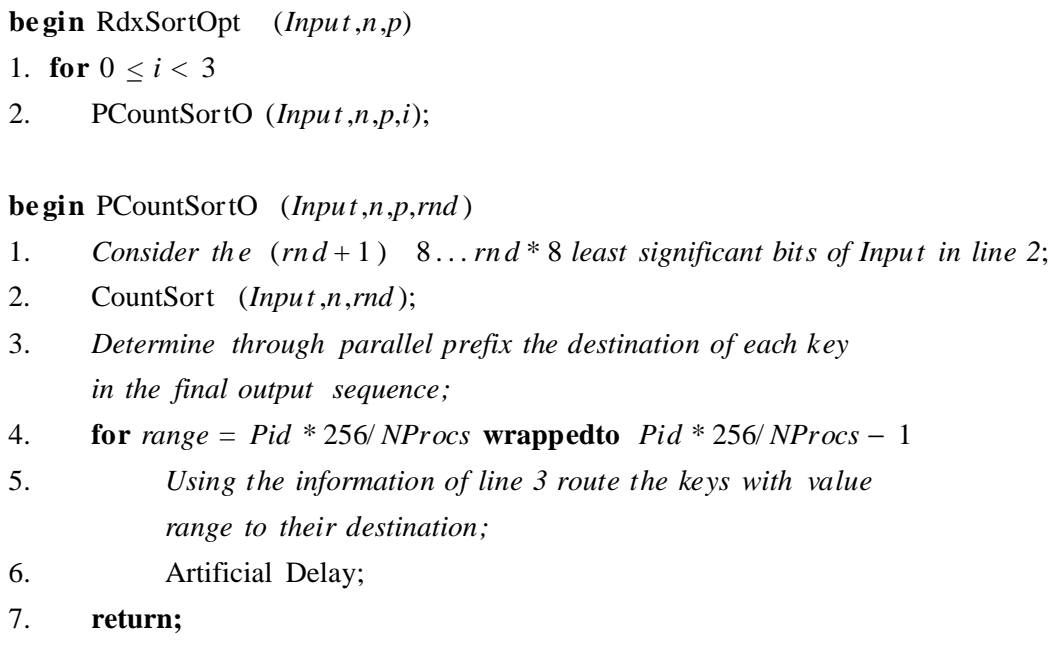

Fig. 10. Procedure RDXSORTOPT.

configurations of the cluster are reported in Figs. 2 and 3 . Note that $1 \mathrm{M}=1024000$. Problem size is the number of integers that are evenly distributed over all the processors. We make sure that the same input set is sorted for increasing $p$. The input consists of uniformly at random generated unsigned 32-bit integers. The sequential time for $n=32 \mathrm{M}$ is indicated by an asterisk because the indicated value is an estimate of actual CPU time. This is because the setup of our cluster (memory limited to $128 \mathrm{Mbytes}$ ) caused the sequential algorithm to swap and its effective running time was several minutes even though CPU time was substantially less. For the same reason, some entries in the tables are left empty; some limited swapping affected the time of RDXSORT and RDXSORTOPT in LAM MPI for the SMP configuration for $n=32 \mathrm{M}$.

One-sided communication was at least $50 \%$ better than two-sided communication. Because of the performance of the send-based implementations we only report figures for put-based implementations in LAM MPI. The enhanced algorithm did not offer any improvements. It seems that software based message combining may be counter productive or messages become so large that they can not be dealt with efficiently by the communication library. Even if BSPlib offers some elementary message combining, this was not used; it is in practice slower than regular communication. Therefore figures for the enhanced algo- 
rithm are not reported either. RDXSORTOPT made marginal difference in the PUB-Library and BSPlib and its contributions were more noticeable in LAM MPI. In non-SMP configurations, BSPlib was a clear winner over LAM MPI, with the PUB-Library suffering considerable degradation in performance for processor sizes $p>2$. In SMP configurations, BSPlib had a slight advantage in the performance of RDXSORT over LAM MPI. When RDXSORTOPT was tested the advantage was reversed. The PUB-Library was a distant third.

We note that maximum observed speedup for a nonSMP configuration was 6.45 for BSPlib and 6.14 for LAM MPI ( $p=16, n=32 \mathrm{M}$ ), and for SMP configurations it was 4.43 for BSPlib and 4.70 for LAM MPI ( $p=16$ and $n=32 \mathrm{M}$ ). These figures are substantially smaller than general purpose parallel sorting algorithms such as those reported in $[12,11]$ which, if executed on the same platform give more than twice the indicated speedup figures. This is understandable, however, for parallel radix-sort. Although the sequential algorithm has performance $4 n r$ (the 4 is the number of rounds of Count-Sort) and $r$ varies between $1 / 2$ microseconds per integer for $n=4 \mathrm{M}$ to $1 / 4$ for $n=32 \mathrm{M}$, the parallel time includes a $4 \mathrm{rn} / \mathrm{p}$ term for parallel computation and a $4(n / p) g$ term for parallel communication, where $g$ is the cost of a total-exchange of size $n / p$. The lowest value for $g$ for $p=16$ from Fig. 6 is 0.59 and 0.93 for BSPlib and LAM MPI in non-SMP and SMP configurations respectively. This is two to four times as much as the $r$ for $n=32 \mathrm{M}$. Therefore the speedups we expect are between $p / 3$ and $p / 5$ and these are the figures we got. The speedup figures provided by the SMP configurations were on the average $30 \%$ lower than the figures of the non-SMP configurations. This indicates that parallel radix-sort is not as suitable as matrix multiplication for SMP PC clusters.

\section{Conclusion}

We made a case for remote memory access as the effective way to program a parallel computer by proposing a robust programmatic framework that supports RMA in a library independent, simple, intuitive, and portable way using the $\mathrm{C}$ programming language. We claimed that if one uses our approach the parallel code one writes will run transparently not only under MPI-2 RMA enabled libraries including LAM MPI and Critical Software's WMPI but also on bulk-synchronous parallel libraries such as BSPlib and PUB-Library thus making one's code library independent as well. We supported our case by implementing using the proposed framework three sets of benchmark quality parallel programs using one-sided communication. Variants of some of these program using two-sided communication were also implemented under LAM MPI. We then examined the performance of these programs on a LINUX-based PC cluster under three different RMA enabled libraries: LAM MPI, BSPlib and PUB-Library, and under LAM MPI for two-sided communication.

We draw some interesting conclusions first for the case of one-sided communication. In the As suite, there was some noticeable deterioration of performance in LAM MPI for put-based compared to send-based communication, which is only natural since the former is built on top of the latter. One-sided communication in BSPlib outperforms one-sided or two-sided communication under LAM MPI; the same can be said for PUB-Library for small $h<4 \mathrm{~K}$, as PUB-Library seems to be not very well tuned.

In the Mult suite, however, the put-based implementations were as good as the send-based ones under LAM MPI; surprisingly enough there was not much difference between put-based and get-based implementations. In BSPlib and PUB-Library, however, these differences were quite noticeable. One-sided communication through BSPlib primarily, and PUB-Library secondarily, outperform two-sided communication. In the $\mathrm{Rdx}$ suite, the put-based implementations were substantially faster than the send-based ones in LAM MPI; for this reason we have not included results for the latter ones. In conclusion, our experiments support the case for one-sided communication. RMA is easier to incorporate in parallel programs, it can be quite efficiently implemented if the overall performance of BSPlib could serve as an indicator, and overall, it is quite well implemented in complex libraries such as LAM MPI even compared to the two-sided communication primitives.

In addition, we make some interesting observations about the performance of the three communication libraries tested. It seems that LAM MPI exhibits the most consistent behavior among the three, even though it may not be the fastest of the three libraries. For the communication patterns of parallel radix-sort the PUB-Library exhibits perhaps an erratic and inconsistent behavior, a possible indication that is probably not fine-tuned in a scalable way. In the As suite of tests, BSPlib and PUB-Library were more efficient than LAM MPI; BSPlib had quite inconsistent default performance that could be improved, however, substantially by setting appropriate values to some communica- 
Table 2

RDXSORT and RDXSORTOPT speedup figures on an non-SMP PC cluster

\begin{tabular}{|c|c|c|c|c|c|c|c|c|c|}
\hline \multirow{3}{*}{ Size } & \multirow{3}{*}{$\begin{array}{c}\text { Time } \\
p=1\end{array}$} & \multicolumn{8}{|c|}{ Non SMP Configuration } \\
\hline & & \multicolumn{4}{|c|}{ RDXSORT } & \multicolumn{4}{|c|}{ RDXSORTOPT } \\
\hline & & $p=2$ & $p=4$ & $p=8$ & $p=16$ & $p=2$ & $p=4$ & $p=8$ & $p=16$ \\
\hline & & \multicolumn{8}{|c|}{ Speedup Results for PUB-Library } \\
\hline $4 \mathrm{M}$ & 2.13 & 0.62 & 0.75 & 0.94 & 1.20 & 0.60 & 0.74 & 0.95 & 1.10 \\
\hline $8 \mathrm{M}$ & 4.30 & 0.61 & 0.77 & 1.01 & 1.76 & 0.61 & 0.74 & 1.02 & 1.60 \\
\hline $16 \mathrm{M}$ & 16.32 & 1.14 & 1.47 & 2.11 & 2.98 & 1.15 & 1.46 & 2.07 & 2.81 \\
\hline \multirow[t]{2}{*}{$32 \mathrm{M}$} & $32.64^{*}$ & - & 1.47 & 2.21 & 3.21 & - & 1.50 & 2.16 & 3.22 \\
\hline & & \multicolumn{8}{|c|}{ Speedup Results for BSPlib } \\
\hline $4 \mathrm{M}$ & 2.13 & 0.73 & 1.14 & 1.75 & 2.96 & 0.73 & 1.15 & 1.72 & 2.88 \\
\hline $8 \mathrm{M}$ & 4.30 & 0.73 & 1.17 & 1.81 & 3.14 & 0.73 & 1.17 & 1.81 & 3.14 \\
\hline $16 \mathrm{M}$ & 16.32 & 1.39 & 2.24 & 3.45 & 6.35 & 1.39 & 2.23 & 3.48 & 6.33 \\
\hline \multirow[t]{2}{*}{$32 \mathrm{M}$} & $32.64^{*}$ & - & 2.24 & 3.51 & 6.26 & - & 2.25 & 3.52 & 6.45 \\
\hline & & \multicolumn{8}{|c|}{ Speedup Results for LAM MPI } \\
\hline $4 \mathrm{M}$ & 2.13 & 0.67 & 0.97 & 1.44 & 1.64 & 0.69 & 1.12 & 1.68 & 2.01 \\
\hline $8 \mathrm{M}$ & 4.30 & 0.59 & 1.04 & 1.60 & 2.15 & 0.59 & 1.12 & 1.89 & 2.35 \\
\hline $16 \mathrm{M}$ & 16.32 & 0.88 & 1.84 & 3.27 & 5.35 & 0.90 & 2.02 & 3.64 & 5.40 \\
\hline $32 \mathrm{M}$ & $32.64^{*}$ & - & 1.39 & 3.20 & 5.75 & - & 1.63 & 3.58 & 6.14 \\
\hline
\end{tabular}

Table 3

RDXSORT and RDXSORTOPT speedup figures on an SMP PC cluster

\begin{tabular}{|c|c|c|c|c|c|c|c|c|c|}
\hline \multirow{3}{*}{ Size } & \multirow{3}{*}{$\begin{array}{c}\text { Time } \\
p=1\end{array}$} & \multicolumn{8}{|c|}{ SMP Configuration } \\
\hline & & \multicolumn{4}{|c|}{ RDXSORT } & \multicolumn{4}{|c|}{ RDXSORTOPT } \\
\hline & & $p=2$ & $p=4$ & $p=8$ & $p=16$ & $p=2$ & $p=4$ & $p=8$ & $p=16$ \\
\hline & & \multicolumn{8}{|c|}{ Speedup Results for PUB-Library } \\
\hline $4 \mathrm{M}$ & 2.13 & 0.79 & 0.69 & 0.81 & 1.75 & 0.79 & 0.74 & 0.83 & 1.43 \\
\hline $8 \mathrm{M}$ & 4.30 & 0.81 & 0.77 & 0.88 & 1.31 & 0.82 & 0.77 & 0.88 & 1.28 \\
\hline $16 \mathrm{M}$ & 16.32 & - & 1.51 & 1.81 & 2.44 & - & 1.48 & 1.81 & 2.47 \\
\hline $32 \mathrm{M}$ & $32.64^{*}$ & - & - & 1.80 & 2.70 & - & - & 1.83 & 2.63 \\
\hline & & \multicolumn{8}{|c|}{ Speedup Results for BSPlib } \\
\hline $4 \mathrm{M}$ & 2.13 & 0.68 & 0.70 & 1.18 & 2.03 & 0.68 & 0.71 & 1.18 & 2.03 \\
\hline $8 \mathrm{M}$ & 4.30 & 0.69 & 0.71 & 1.24 & 2.15 & 0.69 & 0.73 & 1.24 & 2.15 \\
\hline $16 \mathrm{M}$ & 16.32 & - & 1.36 & 2.38 & 4.36 & - & 1.36 & 2.39 & 4.33 \\
\hline $32 \mathrm{M}$ & $32.64^{*}$ & - & - & 2.40 & 4.45 & - & - & 2.43 & 4.43 \\
\hline & & \multicolumn{8}{|c|}{ Speedup Results for LAM MPI } \\
\hline $4 \mathrm{M}$ & 2.13 & 0.75 & 0.83 & 1.09 & 1.30 & 0.77 & 0.79 & 1.30 & 1.72 \\
\hline $8 \mathrm{M}$ & 4.30 & 0.77 & 0.86 & 1.20 & 1.86 & 0.78 & 0.79 & 1.36 & 2.03 \\
\hline $16 \mathrm{M}$ & 16.32 & - & 0.91 & 2.37 & 3.93 & - & 1.05 & 2.49 & 4.53 \\
\hline $32 \mathrm{M}$ & $32.64^{*}$ & - & - & 1.53 & 4.17 & - & - & 1.73 & 4.70 \\
\hline
\end{tabular}

tion control variables. PUB-Library slightly exceeded BSPlib for small processor configurations $(p=4)$ but it did not scale well otherwise. Some surprising results were observed in the Rdx suite. The enhanced algorithm was consistently slower than the straightforward algorithm and timing results for the former were not reported. It seems that program source code-based message combining may not be a good idea for the average programmer. Under LAM MPI the put-based implementations were much faster than the send-based ones; for this reason we did not report results for the latter ones. In the Rdx suite, BSPlib was more efficient than LAM MPI and at least 50\% more efficient than PUB-Library. It is for this reason that we conclude that the PUB-Library may not be properly fine-tuned.

\section{Acknowledgements}

The work of the authors was supported in part by the National Science Foundation under NSF MRI grant EIA-9977508.

\section{References}

[1] G. Bilardi, K.T. Herley, A. Pietracaprina, G. Pucci and P. Spirakis, $B S P$ vs. $\log P$, in Proceedings of the 8-th ACM Symposium on Parallel Algorithms and Architectures, ACM Press, 1996, pp. 25-32.

[2] O. Bonorden, B. Juurlink, I. von Otte and I. Rieping, The Paderborn University BSP (PUB) Library, Parallel Computing 29 (2003), 187-207. 
[3] H.T. Cormen, C.E. Leiserson and L.R. Rivest, Introduction to Algorithms, MIT Press, 1990.

[4] SHMEM Library Ready Reference, Cray Inc.

[5] Critical Software Inc, WMPI.

[6] D.E. Culler, R. Karp, D. Patterson, A. Sahay, K.E. Schauser, E. Santos, R. Subramonian and T. von Eicken, LogP: Towards a Realistic Model of Parallel Computation, in Proceedings of the Fourth ACM SIGPLAN Symposium of Principles and Practice of Parallel Programming, San Diego, CA, ACM Press, 1993, pp. 1-12.

[7] F. Dehne A. Fabri and A. Rau-Chaplin, Scalable Parallel Geometric Algorithms for Coarse Grained Multicomputers, in Proceedings of ACM Symposium of Computational Geometry, San Diego, CA, ACM Press, 1993, pp. 298-307.

[8] R. Dohmen, Experiences with switching from SHMEM to MPI as communication library, in Proceedings of Sixth European SGI/Cray MPP Workshop Manchester, UK, 7-8 September 2000 .

[9] A. Geist, A. Beguelin, J. Dongarra, R. Manchek, W. Jaing and V. Sunderam, PVM: A Users' Guide and Tutorial for Networked Parallel Computing, MIT Press, Boston, 1994.

[10] A.V. Gerbessiotis and S.Y. Lee, Remote memory access: A case for portable, efficient and library independent parallel programming Technical Report CS-03-12, CS Department, New Jersey Institute of Technology.

[11] A.V. Gerbessiotis and L.G. Valiant, Direct bulk-synchronous parallel algorithms, Journal of Parallel and Distributed Computing 22 (1994), 251-267.

[12] A.V. Gerbessiotis and C.J. Siniolakis, Deterministic sorting and randomized median finding on the BSP model, in Proceedings of the 8-th ACM Symposium on Parallel Algorithms and Architectures, Padova, Italy, June 1996, pp. 223-232.

[13] A.V. Gerbessiotis, Architecture Independent Parallel Algorithm Design: Theory vs Practice, Future Generation Com- puter Systems 18 (2002), 573-593.

[14] A.V. Gerbessiotis, http://www.cs.njit.edu/ alexg, July 2003.

[15] P.B. Gibbons, Y. Matias and V. Ramachandran, The queueread queue-write asynchronous PRAM model, in Proceedings of the 9-th ACM Symposium on Parallel Algorithms and Architectures, ACM Press, 1997, pp. 72-83.

[16] J.M.D. Hill, W. McColl, D.C. Stefanescu, M.W. Goudreau, K. Lang, S.B. Rao, T. Suel, T. Tsantilas and R. Bisseling, BSPlib: The BSP Programming Library, Parallel Computing 24(14) (1998), 1947-1980.

[17] LAM/MPI Parallel Computing, http://www.lam-mpi.org.

[18] G. Luecke and W. Hu, Evaluating the Performance of MPI-2 One-Sided Routines on a Cray SV1, Technical Report, Iowa State University, December 2002.

[19] G.R. Luecke, S. Spanoyannis and M. Kraeva, The Performance and Scalability of SHMEM and MPI-2 One-Sided Routines on a SGI Origin 2000 and a Cray T3E-600, Concurrency and Computation: Practice and Experience, to appear.

[20] G.R. Luecke, S. Spanoyannis and J. Coyle, The Performance of MPI Derived Types on a SGI Origin 2000, a Cray T3E900, a Myrinet Linux Cluster and an Ethernet Linux Cluster, Manuscript, 2001.

[21] R. Miller, A library for Bulk-Synchronous Parallel program ming, in Proceedings of the BCS Parallel Processing Specialist Group workshop on General Purpose Parallel Computing, 1993.

[22] MPICH Parallel Computing, //http://www-unix.mcs.anl.gov/ $\mathrm{mpi} / \mathrm{mpich} /$.

[23] D.B. Skillicorn, J.M.D. Hill and W.F. McColl, Questions and Answers about BSP, Scientific Programming 6 (1997), 249274.

[24] L.G. Valiant, A bridging model for parallel computation, Communications of the ACM 33(8) (August 1990), 103-111. 

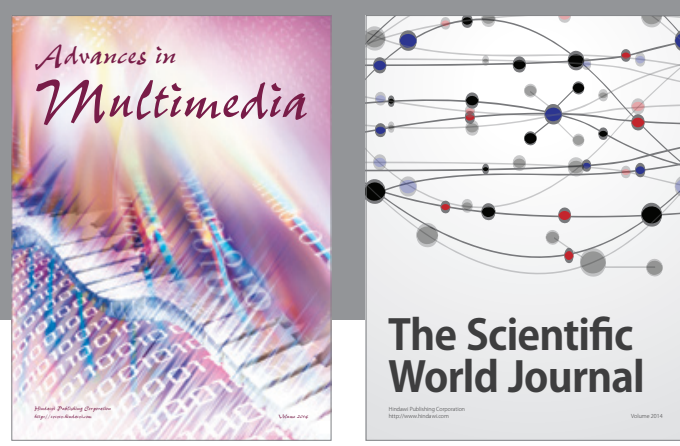

The Scientific World Journal
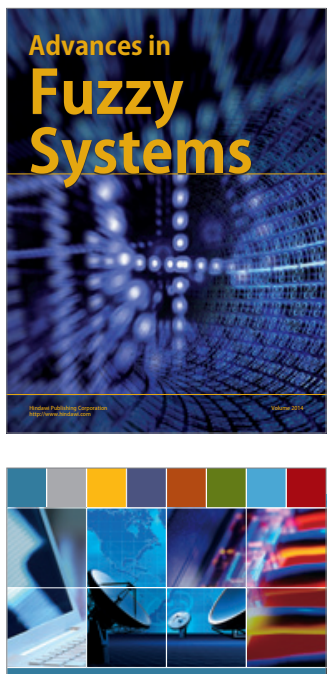

Computer Networks and Communications
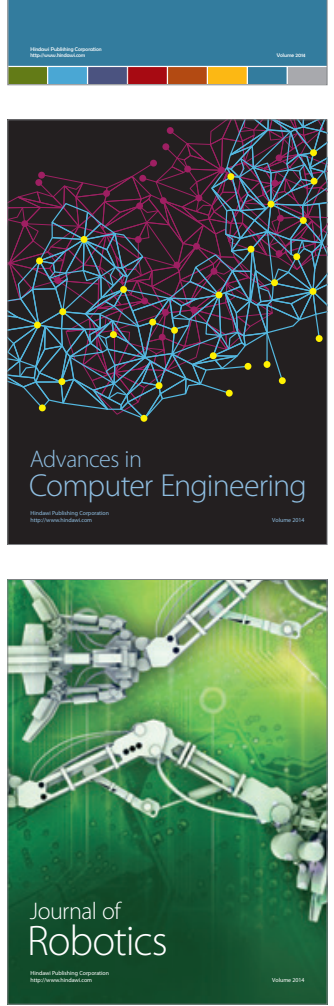
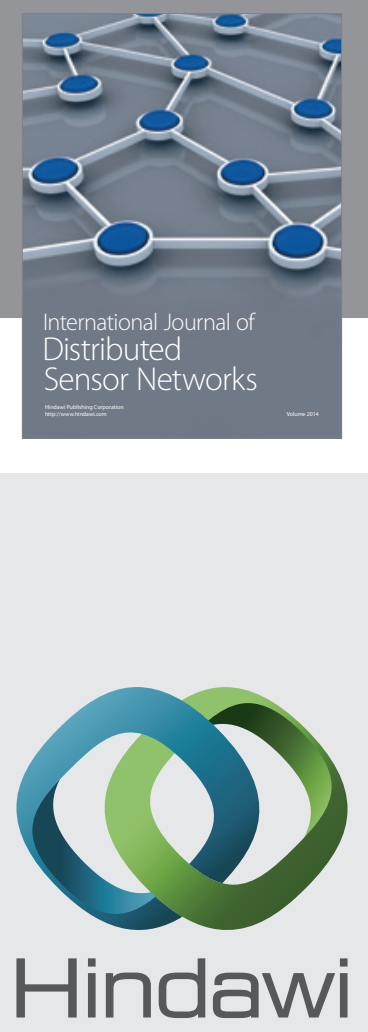

Submit your manuscripts at

http://www.hindawi.com
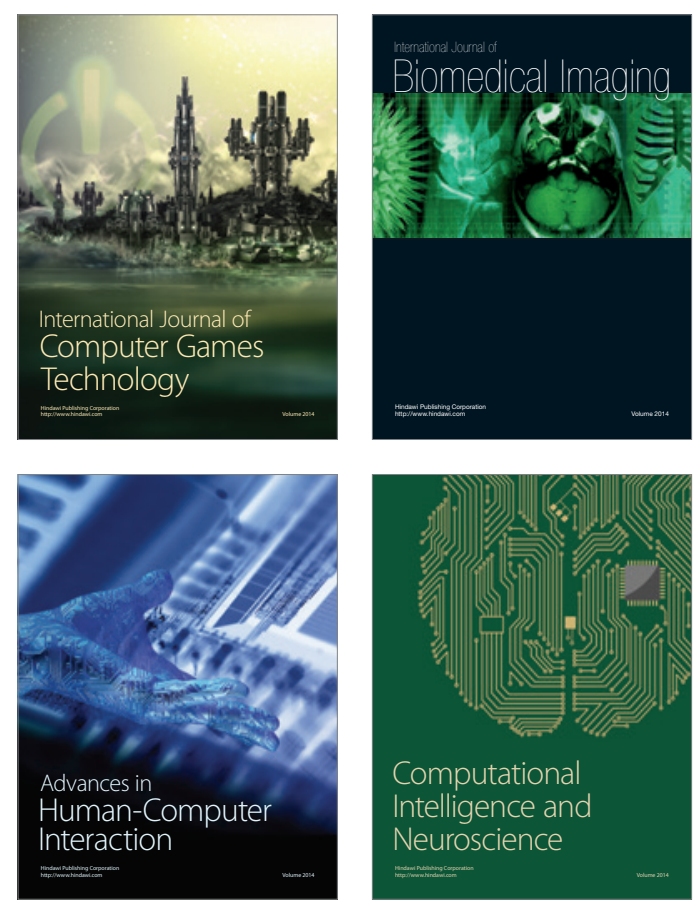
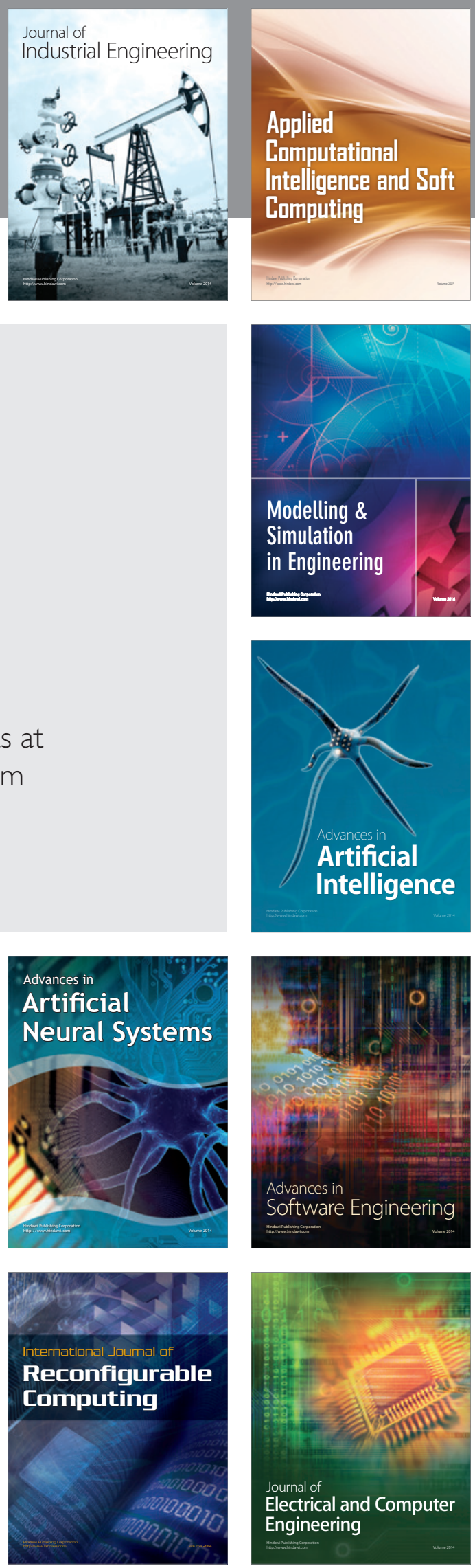